\title{
Recurrent female adnexal tumor of probable Wolffian origin treated with debulking surgery, imatinib and paclitaxel/carboplatin combination chemotherapy: A case report
}

\author{
AKIHIKO WAKAYAMA ${ }^{1}$, HIROFUMI MATSUMOTO ${ }^{2}$, HAJIME AOYAMA $^{2}$, MASANAO SAIO $^{2}$, \\ AYUMI KUMAGAI ${ }^{1}$, TAKUMA OOYAMA ${ }^{1}$, MORIHIKO INAMINE ${ }^{1}$ and YOICHI AOKI $^{1}$ \\ ${ }^{1}$ Department of Obstetrics and Gynecology, Graduate School of Medicine, University of The Ryukyus; \\ ${ }^{2}$ Department of Pathology, University of The Ryukyus Hospital, Okinawa 903-0215, Japan
}

Received May 18, 2016; Accepted January 13, 2017

DOI: $10.3892 / 01.2017 .5874$

\begin{abstract}
In view of the small number of recurrent and metastatic cases of female adnexal tumors of probable Wolffian origin (FATWO), there is a distinct lack of evidence to support recommendations regarding treatment. In the present study, a 37-year-old female was diagnosed with a left adnexal tumor and underwent a left salpingo-oophorectomy (SO). The tumor was found to arise from the left tubal fimbria and extend to the posterior leaf of the broad ligament. Pathological examination identified a number of solid, tubular and cystic architectures, with areas of hyalinization, hemorrhage and necrosis. Immunohistochemistry detected that the tumor cells were positive for cytokeratin (CK)7, vimentin, inhibin and calretinin, and negative for CK20, epithelial membrane antigen, and the estrogen and progesterone receptors. Thus, the patient was diagnosed with FATWO. Following 15 months, the patient developed recurrent tumors and subsequently underwent a total abdominal hysterectomy, a right $\mathrm{SO}$ and extirpation of the disseminated tumors, but with incomplete debulking. The tumor cells were immunoreactive for KIT. As there are a limited number of treatment options and few reported cases, limiting the therapy recommendations, imatinib mesylate was administered for 6 months and the tumors were temporarily stabilized for 4 months until the disease progressed. The patient underwent a further debulking surgery that did not achieve complete debulking and was subsequently administered a combination chemotherapy of paclitaxel and carboplatin. This regimen resulted in an almost complete response after 10 cycles. The tumors continued to decrease in size, and the tumors in the left side
\end{abstract}

Correspondence to: Professor Yoichi Aoki, Department of Obstetrics and Gynecology, Graduate School of Medicine, University of The Ryukyus, 207 Uehara Nishihara, Okinawa 903-0215, Japan E-mail: yoichi@med.u-ryukyu.ac.jp

Key words: female adnexal tumor of probable Wolffian origin, recurrence, c-kit, imatinib, paclitaxel/carboplatin of the Douglas pouch and the right para-colic gutter were no longer detectable. To the best of our knowledge, the current study is the first to report carboplatin and paclitaxel combination therapy demonstrating a response of this degree to recurrent FATWO following the failure of imatinib treatment. However, surgical debulking remains the most effective treatment for FATWO when is it is possible. The precise role of chemotherapy, radiotherapy and molecular-targeting therapy in the treatment of recurrent or metastatic FATWO remains to be elucidated, and therefore, novel strategies to overcome this disease must be prioritized.

\section{Introduction}

Kariminejad and Scully (1) first reported the occurrence of female adnexal tumors of probable Wolffian origin (FATWOs) in 1973, they regarded it as a non-malignant lesion. This rare type of neoplasm typically arises from the persisting remnants of the mesonephric duct following its natural degeneration (1). There are numerous characteristics associated with the possibility of a Wolffian origin, including localization to the broad ligament, where Wolffian remnants are typically found $(2,3)$, and the lack of resemblance to other tumors originating from Müllerian derivatives. The age at diagnosis ranges from 18-81 years, with a mean age of 50 years. Preoperative diagnosis of FATWO is difficult due to the rarity of the disease and the limited literature available. There are no comprehensible recommendations regarding its preoperative workup and optimal treatment (4). FATWOs typically comprise of smallto medium-sized cells that may be ciliated, demonstrating minimal atypia and a number of mitotic figures arranged in a sieve-like microcystic trabecular diffuse pattern (5). The tumor cells are cuboidal or flat with indistinct cytoplasm and bland nuclei and somewhat resemble the cells seen in the remnants of the Wolffian duct (5). Further evidence of the origin of FATWOs is demonstrated by the positive immunoreactivity of these rare tumors to pan-cytokeratin (CK), CAM 5.2 CK antibody, CK7 and vimentin; however, negative immunoreactivity was identified for estrogen receptor (ER), progesterone receptor $(\mathrm{PR})$, epithelial membrane antigen (EMA) and CD20 (6,7). 
Although FATWO tumors typically have been demonstrated to have low malignant potential, with the majority of tumors diagnosed as benign, they have the potential to recur and a number of cases have metastasized (4,8-10). A previous study reported that $20 \%$ of cases are associated with an adverse outcome (6), and that recurrences are treated with a number of distinct of chemotherapy and radiation therapies. As these cases are rare, there are no recommendations, to the best of our knowledge, regarding the initial evaluation and diagnosis, effective treatment, follow-up time period and adjuvant or salvage therapies.

The current study analyzes the case of a 37-year-old female with recurrent FATWO and the clinical, morphological and immunohistochemical observations of this patient, including $c$-kit gene mutational analysis and potential treatment options. Written informed consent was obtained from the patient for the publication of the present study.

\section{Case report}

A 37-year-old primiparous woman without any prior medical history was presented to Naha Municipal Hospital (Naha, Japan) with severe lower abdominal pain in December 2012. Ultrasound examination identified a solid and cystic adnexal mass on the left side, and the patient was referred to University of the Ryukyus Hospital (Okinawa, Japan) in January 2013. Pelvic examination identified a tender mobile tumor in the Douglas pouch. A computed tomography (CT) scan confirmed the presence of a twisted, well-demarcated, multilocular tumor with an irregularly enhancing capsule, implicating the potential for malignancy. The tumor was $70 \times 40 \mathrm{~mm}$ in size and had a number of ascites. T2-weighted magnetic resonance imaging identified the mass as a well-defined ovoid structure associated with a mixture of low- and high-intensity signals, excluding the ovaries (Fig. 1). Serum tumor markers were within normal limits [cancer antigen (CA)-125, $35 \mathrm{IU} / \mathrm{ml}$; carcinoembryonic antigen, $2 \mathrm{ng} / \mathrm{ml}$; CA 19-9, $23 \mathrm{IU} / \mathrm{ml}]$.

The patient was diagnosed with a possible left tubal carcinoma and underwent a laparotomy in February 2013. During the surgery, the mass was identified to have arisen from the left tubal fimbria and extended to the posterior leaf of the broad ligament. The tumor was distinct from the left ovary and was twisted four times. There were no macroscopic intra-abdominal peritoneal implants, whereas micropapillary implants $(<5 \mathrm{~mm})$ were identified on the left ovary. The patient underwent left salpingo-oophorectomy (SO), pelvic lymph node sampling and omental biopsy, and the tumor was resected.

Macroscopic findings of the mass identified a tumor of uneven surface weighing $175 \mathrm{~g}$ and measuring $7 \times 6 \mathrm{~cm}$. The tumor was partially cystic with hemorrhagic areas, and had gray-white and yellow tissue evident at the cut surface (Fig. 2). Pathological examination, in which the tissue sample was fixed in $10 \%$ neutral buffered formalin for $24 \mathrm{~h}$ at room temperature, $3-\mu \mathrm{m}$ sections were cut from formalin-fixed paraffin-embedded tissue blocks, and stained with hematoxylin-eosin, which identified a number of solid, tubular and cystic architectures, with areas of hyalinization, hemorrhage and necrosis. Tubular glands containing an eosinophilic substance were closely packed and outlined by

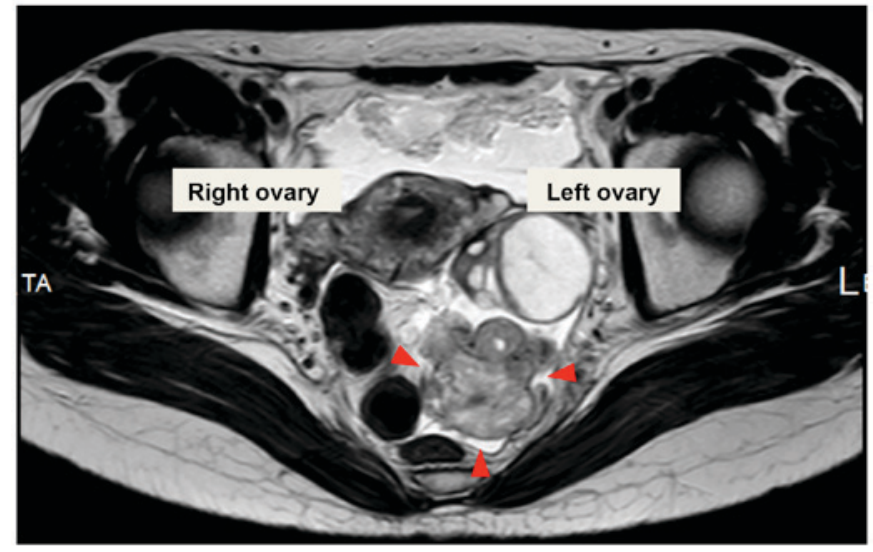

Figure 1. T2-weighted image of magnetic resonance imaging identifying a well-defined, ovoid mass with a mixture of low- and high-intensity signals (arrowheads) residing in regions separate to the ovaries.

a well-defined basement membrane. The tubules were lined with low cuboidal epithelial cells containing uniform round nuclei with mild atypia (Fig. 3). The tumor was comprised of epithelioid and spindle cells growing in a mixture of cribriform, trabecular or solid patterns (11). Neoplastic cells were also identified in ascites and implants on the left ovarian surface. Sections (3- $\mu \mathrm{m}$ thick) were cut from formalin-fixed paraffin-embedded tissue blocks. Immunohistochemistry was performed for calretinin, CK7, CK20, c-kit, epithelial membrane antigen, ER, inhibin, PR, and vimentin using Bench Mark XT (Ventana Medical Systems, Inc., Tucson, AZ, USA) autostainer. Slides were blocked using the Endogenous Biotin Blocking kit (cat. no. 760-050; Ventana Medical Systems, Inc.) for $20 \mathrm{~min}$, Antibodies that were used for this analysis, including the catalog number, dilution and supplier details, the length and temperature of any incubation steps are presented in Table I. Slides were subsequently incubated with a pre-diluted Discovery Universal secondary antibody cocktail (cat. no. 760-4205; Ventana Medical Systems, Inc.) for $32 \mathrm{~min}$.

Immunohistochemical analysis demonstrated that tumor cells were positive for CK7, vimentin, inhibin and calretinin, and negative for CK20, epithelial membrane antigen and ER/PR. The patient was diagnosed with FATWO and followed up with a CT of the abdomen and pelvis every 6 months.

In July 2014, a CT scan of the abdomen and pelvis revealed multiple peritoneal implants. A total abdominal hysterectomy, a right $\mathrm{SO}$ and extirpation of the disseminated tumors were performed, but did not achieve complete debulking. Upon pathological examination, the implant tumor cells exhibited irregular nuclei and clear nucleoli, and were closely proliferated to form irregular glands. Furthermore, the mitotic rate was higher compared with that at the time of primary tumor diagnosis in February 2013. At 6 weeks following the second surgical intervention, a CT scan identified an increase in disseminated tumors in the left side of the Douglas pouch, the right para-colic gutter and the hepatorenal fossa. These tumor cells were immunoreactive for KIT (Fig. 4). Sequencing analysis of exons 9, 11, 13 and 17 of the $c$-kit gene, and exons 12 and 18 of the platelet-derived growth factor receptor (PDGFR) gene $(12,13)$ did not yield any mutational alterations. 
Table I. List of primary antibodies used.

\begin{tabular}{|c|c|c|c|c|c|}
\hline Antibody & Clone & $\begin{array}{l}\text { Supplier and } \\
\text { catalogue no. }\end{array}$ & Dilution & Antigen retrieval & Supplier details \\
\hline Calretinin & $\begin{array}{c}\text { DAK } \\
\text { Calret1 }\end{array}$ & Dako, M7245 & $\mathrm{x} 80$ & $\begin{array}{l}\mathrm{CC} 2 \text {, performed on } \\
\text { autostainer, } 95^{\circ} \mathrm{C}, 60 \mathrm{~min}\end{array}$ & $\begin{array}{l}\text { Dako, North America, Inc., } \\
\text { Carpinteria, CA, USA }\end{array}$ \\
\hline CK7 & RN7 & $\begin{array}{c}\text { Novocastra, } \\
\text { NCL-L-CK7-560 }\end{array}$ & $\mathrm{x} 160$ & $\begin{array}{l}\mathrm{CC} 1, \text { performed on } \\
\text { autostainer, } 95^{\circ} \mathrm{C}, 60 \mathrm{~min}\end{array}$ & $\begin{array}{l}\text { Leica Microsystems, Inc., } \\
\text { Buffalo Grove, IL, USA }\end{array}$ \\
\hline CK20 & Ks20.8 & Leica, NCL-L-CK20 & $\times 50$ & $\begin{array}{l}\mathrm{CC} 1, \text { performed on } \\
\text { autostainer, } 95^{\circ} \mathrm{C}, 60 \mathrm{~min}\end{array}$ & $\begin{array}{l}\text { Leica Microsystems, Inc., } \\
\text { Buffalo Grove, IL, USA }\end{array}$ \\
\hline c-kit & $\begin{array}{c}\text { Rabbit } \\
\text { polyclonal }\end{array}$ & Dako, A4502 & $\mathrm{x} 400$ & $\begin{array}{l}\mathrm{CC} 1 \text {, performed on } \\
\text { autostainer, } 95^{\circ} \mathrm{C}, 60 \mathrm{~min}\end{array}$ & $\begin{array}{l}\text { Dako, North America, Inc., } \\
\text { Carpinteria, CA, USA }\end{array}$ \\
\hline EMA & E29 & Dako, M0613 & $\mathrm{x} 100$ & None & $\begin{array}{l}\text { Dako, North America, Inc., } \\
\text { Carpinteria, CA, USA }\end{array}$ \\
\hline ER & SP1 & Ventana, 790-4324 & Pre-diluted & $\begin{array}{l}\mathrm{CC} 1 \text {, performed on } \\
\text { autostainer, } 95^{\circ} \mathrm{C}, 60 \mathrm{~min}\end{array}$ & $\begin{array}{l}\text { Roche Diagnostics, Basel, } \\
\text { Switzerland }\end{array}$ \\
\hline Inhibin & $\mathrm{R} 1$ & Serotec, MCA951S & $x 50$ & $\begin{array}{l}\mathrm{CC} 1, \text { performed on } \\
\text { autostainer, } 95^{\circ} \mathrm{C}, 60 \mathrm{~min}\end{array}$ & $\begin{array}{l}\text { Funakoshi, Co., Ltd., } \\
\text { Tokyo, Japan }\end{array}$ \\
\hline PR & $1 \mathrm{E} 2$ & Ventana, $790-2223$ & Pre-diluted & $\begin{array}{l}\mathrm{CC} 1 \text {, performed on } \\
\text { autostainer, } 95^{\circ} \mathrm{C}, 60 \mathrm{~min}\end{array}$ & $\begin{array}{l}\text { Roche Diagnostics, Basel, } \\
\text { Switzerland }\end{array}$ \\
\hline Vimentin & V9 & Ventana, 790-2917 & Pre-diluted & $\begin{array}{l}\mathrm{CC} 1, \text { performed on } \\
\text { autostainer, } 95^{\circ} \mathrm{C}, 60 \mathrm{~min}\end{array}$ & $\begin{array}{l}\text { Roche Diagnostics, Basel, } \\
\text { Switzerland }\end{array}$ \\
\hline
\end{tabular}

CC1: Antigen retrieval solution (Ventana Medical Systems, Inc., Tucson, AZ, USA; prediluted; pH 8.5). CC2: Antigen retrieval solution (Ventana; prediluted; pH 6.0). CK, cytokeratin; EMA, epithelial membrane antigen; ER, estrogen receptor; PR, progesterone receptor.

Table II. Primer sequence and fragment length for the exons of c-kit and PDGFRs gene.

\begin{tabular}{|c|c|c|c|}
\hline Gene & Exon & Primer sequence & Fragment length (bp) \\
\hline \multirow[t]{8}{*}{ c-kit } & 9 & F, 5'-ATGCTCTGCTTCTGTACTGCC-3' & 256 \\
\hline & & R, 5'-CAGAGCCTAAACATCCCTTA-3' & \\
\hline & 11 & F, 5'-CCAGAGTGCTCTAATGACTG-3' & 235 \\
\hline & & R, 5'-ACCCAAAAAGGTGACATGGA-3' & \\
\hline & 13 & F, 5'-TGCTCAAGCGTAAGTTCCTG-3' & 335 \\
\hline & & R, 5'-GCAAGAGAGAACAACAGTCTGG-3' & \\
\hline & 17 & F, 5'-TGAACATCATTCAAGGCGTA-3' & 327 \\
\hline & & R, 5'-GCAGGACTGTCAAGCAGAGA-3' & \\
\hline \multirow[t]{4}{*}{ PDGFR } & 12 & F, 5'-CCAGTTACCTGTCCTGGTCAT-3' & 183 \\
\hline & & R, 5'-GGAGGTTACCCCATGGAACT-3' & \\
\hline & 18 & F, 5'-AGTGTGTCCACCGTGATCTG-3' & 146 \\
\hline & & R, 5'-GTGTGGGAAGTGTGGACGTA-3' & \\
\hline
\end{tabular}

PDGFR, platelet-derived growth factor receptor; bp, base pair.

For the sequencing analyses, genomic DNA was extracted from $10 \mu \mathrm{m}$ thick, formalin-fixed, paraffin-embedded tissues following deparaffinization. A polymerase chain reaction (PCR) amplification of exons 9, 11, 13 and 17 of the $c$-kit gene, and exons 12 and 18 of the platelet-derived growth factor receptor (PDGFR) gene was performed using the primer sets for PCR as presented in Table II. The PCR products were directly sequenced using an ABI PRISM 310 genetic analyzer (Applied Biosystems, Thermo Fisher Scientific, Inc.), according to the manufacturer's protocol.
As there are limited options for treatment of this condition, $400 \mathrm{mg}$ (orally, once a day) imatinib mesylate (Glivec; Novartis Pharma K.K., Tokyo, Japan), a tyrosine kinase inhibitor with minimal side effects, was administered for 6 months. The Glivec treatment of the patient was approved by the Institutional Review Board of University of the Ryukyus (Okinawa, Japan). Although the tumors were temporarily stabilized (for 4 months), the disease became progressive. In June 2015, the patient underwent a further debulking surgery that did not achieve complete debulking. At 6 weeks following the third 


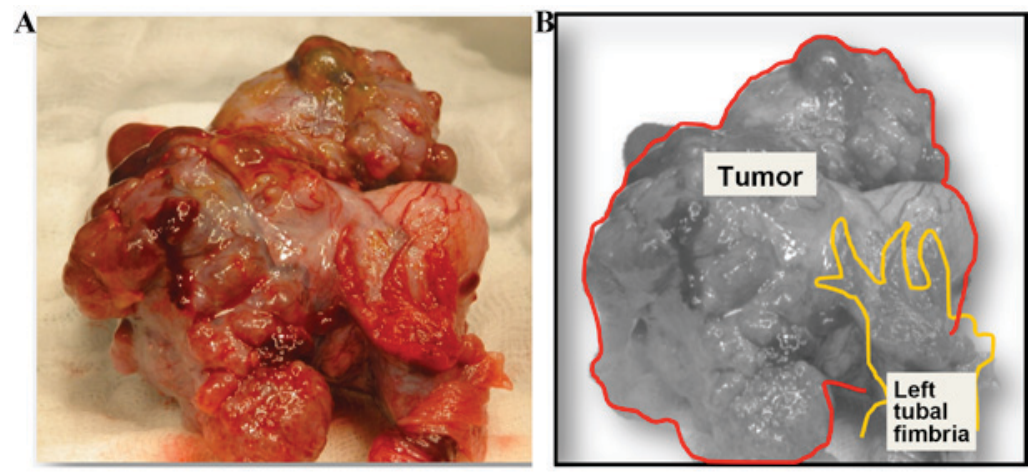

Figure 2. Macroscopic characteristics of the patient's tumor, which had an uneven surface, weighed $175 \mathrm{~g}$ and measured $7 \mathrm{x} 6 \mathrm{~cm}$. (A) The tumor was partially cystic with hemorrhagic areas, with gray-white and yellow tissue visible on the cut surface. (B) The area of the tumor (red line) and the area that is the left tubal fimbria (yellow line).

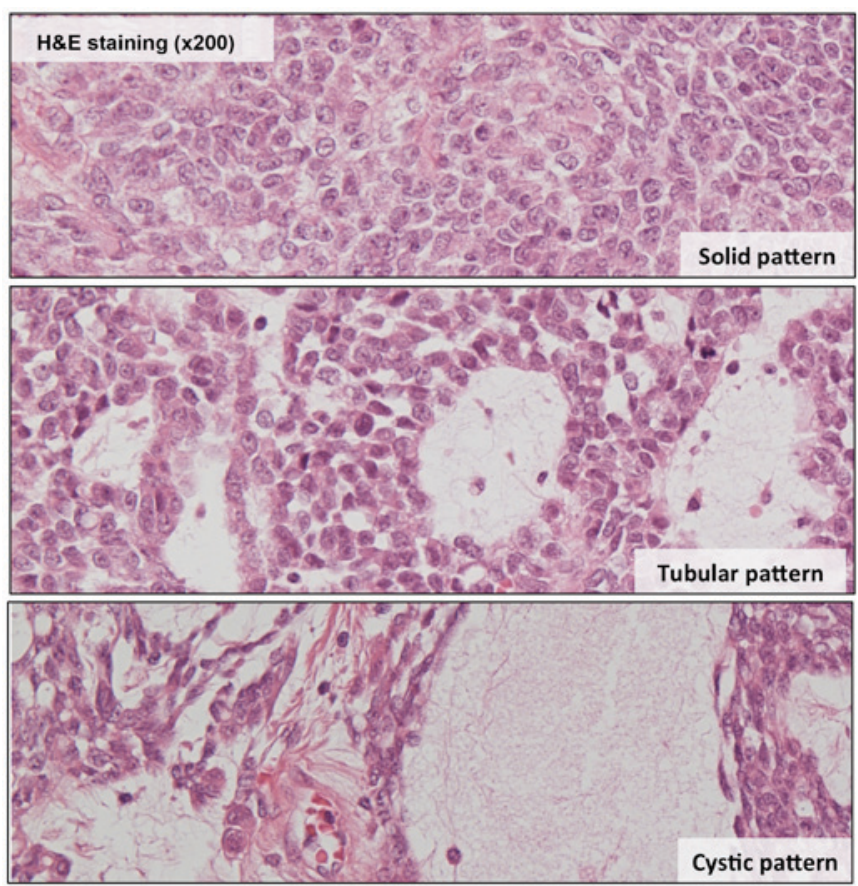

Figure 3. H\&E staining (x200 magnification) demonstrating solid, tubular and cystic architectures. Tubular glands containing an eosinophilic substance were closely packed and outlined by well-defined basement membranes. Tubules were lined with low cuboidal epithelial cells containing uniform round nuclei with mild atypia. H\&E, hematoxylin and eosin.

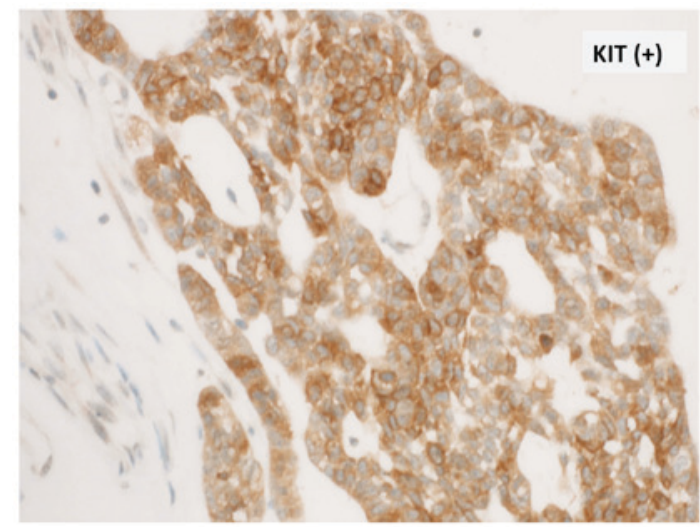

Figure 4. Disseminated tumor demonstrating moderate to weak membrane and cytoplasmic KIT oncogene brown staining in contrast to the absence of staining in the adjacent normal stroma (x400 magnification). surgical intervention, a CT scan identified an increase in the disseminated tumors in the left side of the Douglas pouch, the right para-colic gutter and the hepatorenal fossa (Fig. 5A). The patient was administered a combination chemotherapy of paclitaxel $\left(180 \mathrm{mg} / \mathrm{m}^{2}\right)$ and carboplatin (area under the curve, 5), every 3 weeks. This regimen resulted in a partial response after 6 cycles. With further cycles, the tumors continued to decrease in size, and the tumors in the left side of the Douglas pouch and the right para-colic gutter were no longer detectable after 10 cycles (Fig. 5B). During June, 2016, the patient was undergoing combination chemotherapy treatment.

\section{Discussion}

The current study reports the case of a patient with recurrent FATWO who was treated with debulking surgeries, imatinib mesylate and paclitaxel/carboplatin combination chemotherapy. Tubal malignancy was suspected prior to the primary surgery. However, pathological and immunohistochemical analysis subsequently identified the lesion to be a FATWO. Following conservative surgery, chemotherapy and radiation therapy was not administered and the patient was scheduled for clinical follow-up. Although the majority of FATWO cases are benign, the literature has established that certain tumors have the potential to recur and metastasize. Metastasis and recurrence has been established to occur in 11-20\% of cases and may present within 2 years $(4,8-10)$. The most frequent sites of metastasis observed in cases with FATWO are the liver and the lungs $(1,6)$. In the present patient, the tumor recurred in the form of peritoneal dissemination within 1.5 years of completion of the first treatment. In previous studies, the median recurrence time was reported as 48 months, with a range of 13-96 months, and in certain cases, tumors recurred after extended intervals following diagnosis $(4,9)$. To the best of our knowledge, there are no histological characteristics or biomarkers that associate with tumor outcomes in patients with FATWO.

The treatment options for FATWO tumors remain poorly characterized, as they are rarely reported. However, the therapy with the highest efficacy appears to be complete surgical resection, including a hysterectomy and bilateral adnexectomy $(4,8-10)$. The majority of recurrent tumors have developed in patients that were initially treated with 

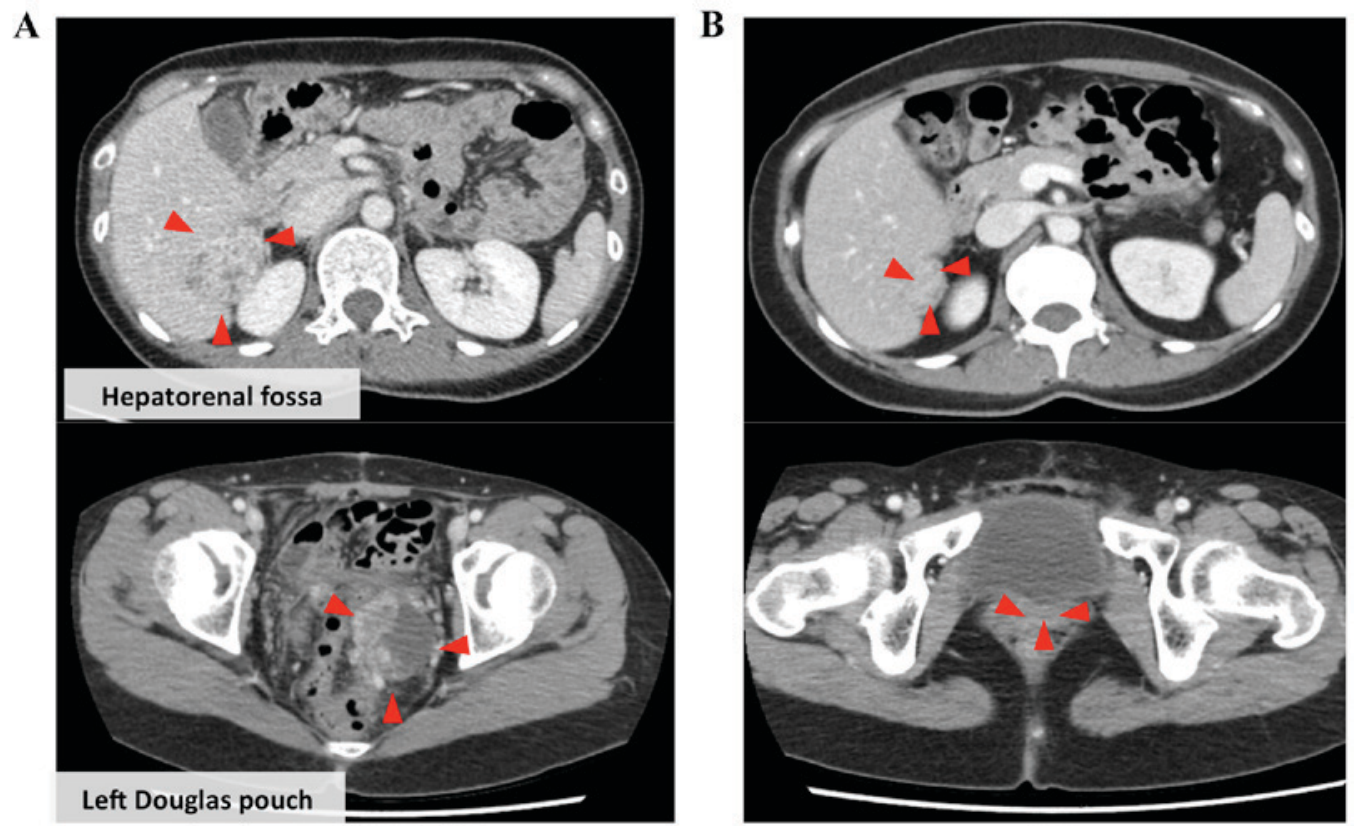

Figure 5. Abdominal and pelvic CT demonstrating that paclitaxel and carboplatin combined chemotherapy resulted in an almost complete response. (A) CT taken prior to paclitaxel and carboplatin treatment, and (B) after 10 cycles of the regimen. Arrowheads indicate the tumors in the hepatorenal fossa (top images) and the left Douglas pouch (bottom images). CT, computed tomography.

tumor resection alone (9). The role of adjuvant chemotherapy or radiation therapy in the treatment of FATWO remains controversial. Therefore, there are limited options for the treatment of recurrent and metastatic disease (6).

For recurrent and metastatic FATWO, molecular-targeted therapy, such as the use of tyrosine kinase inhibitors, may be a potential treatment option. KIT immunoreactivity has been identified in three cases with FATWO. -kit gene analysis was performed in $2 / 3$ of these cases and no mutations were detected by polymerase chain reaction analysis (14-16), as was found in the current study. A Japanese study reported a FATWO case in which the tumor tissues were positive for KIT, as determined using immunohistochemical stain, but in which there were no mutations found in exons 9 or 11 of the $c$-kit gene, suggesting the potential of molecular therapy targeted to KIT (14). A study by Steed et al (15) demonstrated that following initial chemotherapy failure, imatinib mesylate therapy was effective at reducing tumor recurrence size in a patient after 12 weeks of therapy, and the patient achieved complete remission after 10 months of follow-up. This previous study detected positive KIT immunostaining in the patient's recurrent lesions, however, $c$-kit gene analysis was not performed (15). Tumor cells from a patient reported by Syriac et al (16) were immunoreactive for KIT, whereas exons 9, 11, 13 and 17 of the $c$-kit gene, and exons 12 and 18 of the PDGFR gene were not mutated. Despite insufficient previous studies investigating the effect of imatinib mesylate on FATWO tumors, this targeted molecular therapy was administered in this case of recurrent KIT-positive FATWO for 6 months and follow-up was performed at 6-month intervals. However, the patient's prognosis was not reported in this study (16). In the present study of a patient with recurrent KIT-positive protein FATWO, the tumors were temporarily stabilized for 4 months until the disease became progressive. To determine the effectiveness of this therapeutic option, further study is required from multi-center studies.

A number of chemotherapy regimens, including cisplatin/cyclophosphamide, etoposide/ifosfamide/carboplatin, cisplatin/paclitaxel, carboplatin/paclitaxel with leuprolide, and irinotecan alone, have been used to treat recurrent and metastatic FATWO $(4,15,17)$. However, only paclitaxel used in combination with cisplatin induced temporary stabilization of the disease (17). To the best of our knowledge, the current study is the first report of carboplatin and paclitaxel combination therapy yielding a marked response in recurrent FATWO following the failure of imatinib treatment. However, the patient must continue be monitored through follow-up examinations.

In conclusion, as there are a small number of reported recurrent and metastatic FATWO cases, there is a distinct lack of evidence to validate treatment recommendations, and prospective clinical trials are difficult. Surgical debulking is the most effective treatment when it is possible. The precise role of chemotherapy, radiotherapy and molecular-targeting therapy in treating FATWO remains to be elucidated. Therefore, novel strategies to overcome recurrent and metastatic FATWO are required.

\section{Acknowledgements}

The English language service Enago ${ }^{\mathrm{TM}}$ aided in the preparation of the current study manuscript for publication.

\section{References}

1. Kariminejad MH and Scully RE: Female adnexal tumor of probable Wolffian origin. A distinctive pathologic entity. Cancer 31: 671-677, 1973. 
2. Brescia RJ, Cardosa de Almeida PC, Fuller AF Jr, Dickersin GR and Robboy SJ: Female adnexal tumor of probable wolffian origin with multiple recurrences over 16 years. Cancer 56: $1456-1461,1985$

3. Prasad CJ, Ray JA and Kessler S: Female adnexal tumor of wolffian origin. Arch Pathol Lab Med 116: 189-191, 1992.

4. Ramirez PT, Wolf JK, Malpica A, Deavers MT, Liu J and Broaddus R: Wolffian duct tumors: Case reports and review of the literature. Gynecol Oncol 86: 225-230, 2002

5. Daya D, Young RH and Scully RE: Endometrioid carcinoma of the fallopian tube resembling an adnexal tumor of probable wolffian origin: A report of six cases. Int J Gynecol Pathol 11: 122-130, 1992

6. Heatley MK: Is female adnexal tumor of possible wolffian origin a benign lesion? A systematic review of the English literature. Pathology 41: 645-648, 2009.

7. Deen S, Duncan TJ and Hammond RH: Malignant female adnexal tumors of probable Wolffian origin. Int $\mathrm{J}$ Gynecol Pathol 26: 383-386, 2007

8. Sheyn I, Mira JL, Bejarano PA and Husseinzadeh N: Metastatic female adenexal tumor of probable wolffian origin: A case report and review of the literature. Arch Pathol Lab Med 124: 431-434, 2000.

9. Lesin J, Forko-Ilić J, Plavec A and Planinić P: Management of Wolffian duct tumor recurrence without chemotherapy. Arch Gynecol Obstet 280: 855-857, 2009.

10. Heller DS, Kadire B and Cracchiolo B: Malignant female adnexal tumor of probable Wolffian origin: A case report. J Reprod Med 56: 175-177, 2011.
11. Lawrence W, Seidman J, Alvarado-Cabrero I and Wells M: Wolffian tumor. In: Kurman R, Carcangiu M, Herrington S, Young R (eds). WHO Classification of Tumors of Female Reproductive Organs. Geneva: WHO Press, pp117-118, 2014.

12. Antonescu CR, Sommer G, Sarran L, Tschernyavsky SJ, Riedel E, Woodruff JM, Robson M, Maki R, Brennan MF, Ladanyi M, et al: Association of KIT exon 9 mutations with nongastric primary site and aggressive behavior: KIT mutation analysis and clinical correlates of 120 gastrointestinal stromal tumors. Clin Cancer Res 9: 3329-3337, 2003.

13. Do I, Araujo ES, Kalil RK, Bacchini P, Bertoni F, Unni KK and Park YK: Protein expression of KIT and gene mutation of c-kit and PDGFRs in Ewing sarcomas. Pathol Res Pract 203: 127-134, 2007.

14. Harada O, Ota H, Takagi K, Matsuura H, Hidaka E and Nakayama J: Female adnexal tumor of probable wolffian origin: Morphological, immunohistochemical, and ultrastructural study with c-kit gene. Pathol Int 56: 95-100, 2006.

15. Steed H, Oza A, Chapman WB, Yaron M and De Petrillo D Female adnexal tumor of probable wolffian origin: A clinicopathological case report and a possible new treatment. Int J Gynecol Cancer 14: 546-550, 2004.

16. Syriac S, Durie N, Kesterson J, Lele S and Mhawech-Fauceglia P: Female adnexal tumor of probable Wolffian origin (FATWO) with recurrence 3 years postsurgery. Int J Gynecol Pathol 30: 231-235, 2011.

17. Atallah D, Rouzier R, Voutsadakis I, Sader-Ghorra C, Azoury J, Camatte S, Morice P and Duvillard P: Malignant female adnexal tumor of probable wolffian origin relapsing after pregnancy. Gynecol Oncol 95: 402-404, 2004. 